Gericht, bei dem eine von mehreren danach zulässigen Klagen anhängig gemacht worden ist, statt dem Verfahren Fortgang zu geben, auch ohne Antrag des Klägers - ggf. sogar gegen seinen Willen - durch Aktenvorlage eine Gerichtsstandsbestimmung herbeiführen könnte. Aber auch dann, wenn der Kläger - wie hier - vor einem bestimmten Gericht Klage zunächst nur gegen einen von mehreren Beklagten als Streitgenossen eingereicht hat, besteht kein Anlass, dem angerufenen Gericht die Befugnis einzuräumen, von Amts wegen eine Gerichtsstandsbestimmung nach $\$ 36$ Nr. 3 ZPO herbeizuführen. Ist das Gericht der Ansicht, nicht hinsichtlich aller Beklagten für die Klage zuständig $\mathrm{zu}$ sein, kann es den Kläger darauf hinweisen und ihm Gelegenheit geben, einen Antrag auf Bestimmung eines gemeinsamen Gerichtsstands für alle Klagen zu stellen. Dieser Hinweispflicht ist die Einzelrichterin des LG Bad Kreuznach nachgekommen.

Ein Kläger, der die so verlautbarte Rechtsansicht des Gerichts nicht teilt, hat lediglich Anspruch darauf, dass das angerufene Gericht über seine Zuständigkeit eine Entscheidung trifft, die er ggf. mit Hilfe eines Rechtsmittels angreifen kann. Dieser Anspruch darf ihm nicht durch eine von Amts wegen veranlasste Gerichtsstandsbestimmung genommen werden (vgl. BGH, NJW-RR 1991, 767).

Dementsprechend ist der Senat auch nicht befugt, das vorliegende PKH-Beschwerdeverfahren auszusetzen und die Sache dem zur Bestimmung eines gemeinsam zuständigen Gerichts berufenen 4. Zivilsenat des OLG Koblenz vorzulegen. Auch muss nicht entschieden werden, welche
Rechtsfolgen es hat, dass der Antragsteller sein Wahlrecht nach $\$ 35$ ZPO mit der Klageerhebung bei dem LG Bad Kreuznach mit Blickrichtung auf den beklagten Arzt bereits verbraucht hat. Für ihn ist das LG Bad Kreuznach gemäß $\$ \$ 12,13$ ZPO zweifelsfrei zuständig. Das Verfahren nach $\$ 36$ Abs. 1 Nr. 3 ZPO ist darauf gerichtet, ggf. eine zusätzliche Zuständigkeit zu begründen. Es soll aber nicht ein zuständiges Gericht zu einem unzuständigen machen. Daher spricht vieles dafür, dass eine anderweitige Zuständigkeitsbestimmung nicht mehr in Betracht kommt, wenn die Klage bereits bei einem Gericht rechtshängig gemacht worden ist, das für mindestens einen Streitgenossen zuständig ist. Ein Antrag nach $\$ 36$ Abs. 1 Nr. 3 ZPO kann unter Umständen aber auch daran scheitern, dass hier für den bei Prozessbeginn im Bezirk des LG Koblenz wohnhaften K1. wegen der Folgen der behaupteten Behandlungsfehler und -versäumnisse möglicherweise von Anfang an der besondere Gerichtsstand des $\$ 32$ ZPO und damit die Zuständigkeit des LG Koblenz eröffnet war (Ort des Eintritts des schädlichen Erfolgs). Demzufolge könnte $\$ 36$ Abs. 1 Nr. 3 ZPO (am Ende) einschlägig sein.

Auf all das weist der Senat vorsorglich hin, um den K1. nicht $\mathrm{zu}$ einem möglicherweise ebenfalls aussichtslosen Antrag auf Bestimmung eines gemeinsam zuständigen Gerichts zu verleiten.

Dass das LG Bad Kreuznach für die beabsichtigte Klage gegen die Trägerin des Krankenhauses in Z. örtlich nicht zuständig ist, wird von der Beschwerde nicht in Zweifel gezogen.

\title{
REZENSIONEN
}

DOI: $10.1007 / \mathrm{s} 00350-010-2798-7$

\section{Krankenhaushaftung, Organisation, Schadensverhütung und Versicherung. Leitfaden für die tägliche Praxis.}

Herausgegeben von Karl Otto Bergmann u. Hans-Friedrich Kienzle. Deutsche Krankenhaus-Verlagsgesellschaft, Düsseldorf, 3. Aufl. 2010, XIV u. 442 S., kart., $€ 34,00$

Nach Auskunft des Statistischen Jahrbuchs 2009 für die Bundesrepublik Deutschland bestanden im Jahre 2007 hierzulande 2087 Krankenhäuser mit 1.067.287 Beschäftigten, unter ihnen (nach dem Tätigkeitsbericht der BÄK von 2009) 150.644 Ärzte, mit einem Personalkostenaufwand von 41.929.684.000 € für 17.178.573 Fälle; ein Drittel der 39,7 Millionen klinischen Maßnahmen entfiel auf Operationen - ein wahrlich weites und komplexes Feld: in fortwährender Bewegung, für die Stichworte stehen wie Privatisierung, Kostendruck, Fallpauschalen, Qualitäts- und Risikomanagement, Haftpflichtfälle und -risiken, Personalnöte, Verrechtlichung. Das vorliegende Gemeinschaftswerk von nicht weniger als sechzehn Autoren, dessen Vorauflage 2003 erschien, deckt dieses teils schwer zugängliche Gebiet im wesentlichen ab.

Ein großer Vorzug des Buches liegt darin, dass Juristen und Ärzte zusammenwirkten: Anwälte, Kliniker, Verwaltungs- und Versicherungsfachleute, wodurch die Praxisnähe gewährleistet bleibt. Wenn Juristen und Ärzte sich zu den nämlichen Themen äußern, lassen sich Überschneidungen nicht vermeiden; sie sind aber im Interesse umfassender Unterrichtung aus verschiedener, jeweils legitimer Perspektive in Kauf zu nehmen. Wo das Arztrecht sich als Richterrecht

Prof. Dr. iur. Dr. h.c. Adolf Laufs,

Heidelberg, Deutschland darstellt wie etwa ausgeprägt bei der Aufklärungsthematik, kommt es dagegen zu unwillkommenen Wiederholungen, wenn das Buch den Stoff unter Juristen aufteilt in die Kategorien „Grundlagen“ und „Rechtsprechung“"

Das Buch zeichnet sich weiter aus durch umfängliche Informationen zum Management im umfassenden Sinne der Organisation, der Risikominimierung, der Versicherung. Der Leser gewinnt einen starken Eindruck von den zunehmenden, wohl unumgänglichen Verwaltungslasten, auch vom Vordringen wirtschaftlicher Kriterien und ökonomischer Terminologie. Dabei soll der einzelne Patient in seiner Individualität doch immer im Mittelpunkt stehen und der Arzt ,,in den Grenzen zwischen kostenaufwändiger Defensivmedizin und standardunterschreitender Sparsamkeit" (S. 59; dazu neuerdings die Monographie von Johannes Arnade, Kostendruck und Standard, 2010).

Das Werk umfasst acht Teile: Grundlagen der Krankenhausorganisation und -haftung; Organisation des ärztlichen und nichtärztlichen Dienstes (Standard und Haftung, Arbeitsteilung, Dokumentation, Judikatur nach medizinischen Fächern); Patientenaufklärung; Krankenhausverwaltung; Zivil- und Strafverfahren; Krankenhaushaftung und Versicherung; Risk-Management. Wie ein roter Faden durchzieht das Bemühen um die gebotene Krankenhausqualität, um das Vermeiden von Fehlern alle Partien. Es mangelt auch nicht an Auskünften über Arbeitszeit und Nachtdienst (S. 270) und die Fachaufsicht (S. 180f.). Aber es fehlt ein Abschnitt zur Berufsnot vieler Ärzte im Zeichen von Engpässen infolge unzureichender Klinikbudgets und zur Fürsorgepflicht und zur Remonstration ärztlicher und administrativer Vorgesetzter: Ärzte und Pflegedienst haben oft ein Übermaß an Hilfe für die Patienten und an Schreibarbeit zu tragen ein akutes Problem, auch mit rechtlichen Dimensionen.

Die durchweg gut belegte Darstellung kommt dem Leser entgegen durch klare Gliederung und drucktechnische Mittel: Hervorhebung durch Fettdruck, Kasten und Graphiken, ganz im Dienst des ersten Zugriffs. So wird das handliche Gemeinschaftswerk nicht nur Juristen und Verwaltungsleuten, sondern auch Ärzten von Nutzen sein. 